\title{
Aumento della creatininemia a un controllo di routine
}

\author{
Gianna d'Adamo
}

U.O.C. Nefrologia e Dialisi, Ospedale S. Spirito, ASL Roma /E, Roma

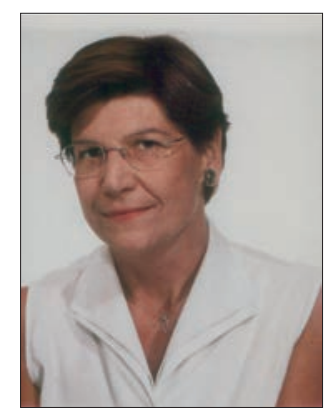

Il controllo della funzione del rene trapiantato è certamente un obiettivo fondamentale - e per il paziente spesso l'unico motivo - dei controlli post-trapianto. Il dosaggio della creatinina sierica è l'indice di funzione renale più semplice $\mathrm{e}$ rapidamente disponibile presso tutti i laboratori ed è l'unico "raccomandato" dalle linee guida che ne suggeriscono anche la periodicità minima: ogni giorno nella prima settimana; due-tre volte/ settimana dalla seconda alla quarta; una volta/settimana nel secondo e terzo mese; ogni due settimane fino al sesto mese; mensilmente fino alla fine del primo anno; e ogni due-tre mesi per tutta la durata del trapianto (1).

Nelle prime settimane la frequenza dei controlli deve essere maggiore perché è maggiore il rischio di alterazioni della funzione renale per cause immunologiche e non immunologiche che si possono trattare in maniera più efficace se vengono evidenziate tempestivamente: rigetto acuto, danno vascolare, stravaso di urina, ostruzione della via urinaria o recidiva precoce della malattia di base (2). I controlli vengono per lo più immediatamente riferiti o eseguiti direttamente dal Centro Trapianti che generalmente provvede anche a gestire l'eventuale complicanza.

Dal quarto mese il rischio di rigetto si riduce e, dopo il primo anno, è modesto nei pazienti che si attengono alla terapia immunosoppressiva prescritta. La maggior parte dei controlli di routine può essere eseguita presso l'ambulatorio di un Centro di Nefrologia, non sede di Centro Trapianti. La collaborazione tra l'ambulatorio di nefrologia e il Centro Trapianti generalmente favorisce il rispetto del programma dei controlli e consente al paziente di avere un nefrologo di riferimento più vicino al proprio domicilio e al corrente della situazione clinica (3).

\section{Indagini di primo livello}

Al riscontro di un incremento della creatininemia in occasione di un controllo programmato, il nefrologo dell'ambulatorio deve orientarsi tra due possibili contesti che richiedono modalità e tempi di indagine radicalmente diversi: danno renale acuto o danno renale cronico?

L'entità dell'incremento della creatinina, l'età del trapianto e il colloquio con il paziente sono gli elementi essenziali per il primo orientamento. Si devono indagare:

- episodi febbrili recenti o in atto, disturbi minzionali, disturbi digestivi o qualunque altra variazione rispetto allo stato di salute precedente;

- entità della diuresi;

- variazioni nell'alimentazione e/o nell'introduzione di liquidi;

- andamento della pressione arteriosa a casa;

- aderenza alla terapia immunosoppressiva prescritta;

- assunzione di nuovi farmaci, anche da banco;

- recente sospensione/aggiunta di farmaci per iniziativa del paziente o prescrizione di un altro medico;

- precedenti infezioni;

- contatti con pazienti infetti.

L'esame obiettivo generale e la misurazione della pressione arteriosa e della frequenza cardiaca consentono di evidenziare o escludere una disidratazione.

Ispezione, palpazione e auscultazione della fossa iliaca sede dell'organo trapiantato non devono essere trascurati. L'ispezione può evidenziare segni di infezione della ferita chirurgica, nel primo periodo post-trapianto. Un soffio vascolare può essere presente in caso di stenosi dell'arteria renale. Il rene può essere dolorabile o aumentato di volume per rigetto acuto, uropatia ostruttiva, nefrite interstiziale acuta o trombosi venosa. Da notare tuttavia che, con le attuali terapie immunosoppressive, la maggior parte degli episodi di rigetto acuto non si accompagnano ai segni clinici classici (febbre, contrazione 
della diuresi e dolorabilità dell'organo).

Stick urinario, esame del sedimento urinario ed esami ematochimici di routine, vanno eseguiti quanto prima, insieme, se possibile, al dosaggio degli immunosoppressori, in particolare dell'eventuale inibitore delle calcineurine.

L'ecografia renale consente di diagnosticare o escludere l'uropatia ostruttiva; l'ecocolordoppler la trombosi arteriosa e venosa.

\section{Danno renale acuto}

In caso di variazioni della funzione renale nel breve termine, le formule per la stima del VFG non presentano vantaggi rispetto al semplice dosaggio della creatininemia; infatti il valore di quest'ultima è, nelle diverse formule, l'unico fattore che può variare significativamente nell'arco di poco tempo. Si parla di danno renale acuto con effettiva riduzione del VFG se, a distanza di 48 ore, l'aumento della creatininemia è $\geq 50 \%$ o comunque $\geq 0,3$ $\mathrm{mg} / \mathrm{dl}(4)$.

Un aumento della creatininemia non riconducibile a disidratazione, uropatia ostruttiva, complicanze vascolari, tossicità da farmaci - non solo da inibitori della calcineurina - deve far pensare a un episodio di rigetto acuto. Infatti, nonostante l'introduzione di nuovi potenti farmaci immunosoppressivi, il rigetto acuto resta una causa importante di disfunzione nel primo anno dopo il trapianto e può manifestarsi a qualsiasi distanza dall'intervento in particolare se viene variata l'immunosoppressione o se viene meno l'aderenza del paziente alla terapia immunosoppressiva.

La maggior parte degli episodi di rigetto acuto è relativamente asintomatica e l'incremento della creatininemia ha sensibilità e specificità limitate e va considerato solo un indicatore per decidere quando eseguire la biopsia del rene trapiantato (5). La diagnosi certa non è infatti possibile in assenza di conferma istologica e la biopsia dovrebbe essere eseguita sempre, a meno che ciò comporti un eccessivo ritardo nell'istituzione della terapia (1). La biopsia viene generalmente eseguita presso il Centro Trapianti, o comunque decisa in accordo con il Centro. Listologia conferma la diagnosi, distingue tipo e gravità del danno e orienta la terapia, ma talora può evidenziare cause di disfunzione renale che peggiorerebbero con la terapia antirigetto o la concomitanza di rigetto acuto e tossicità da inibitori delle calcineurine 0 nefropatia da BKV (6).

Anche la nefrotossicità da inibitori delle calcineurine può essere sospettata solo controllando l'andamento funzionale del trapianto. La ciclosporina e il tacrolimus, tuttora inclusi nella maggior parte dei protocolli terapeutici, possono causare vasocostrizione dell'arteriola afferente e diminuzione del filtrato dose-dipendente e reversibile $(1,2)$. Il livello ematico dell'immunosoppressore è un indicatore utile ma impreciso perché, anche in presenza di tossicità, il valore può essere sovrapponibile a quello dei pazienti con funzione renale stabile. Nell' $1-4 \%$ dei trapiantati renali in terapia con ciclosporina è stata segnalata anche microangiopatia trombotica, non dose-dipendente, verosimilmente su base idiosincrasica, che si manifesta con insufficienza renale acuta con o senza trombocitopenia, anemia emolitica microangiopatica, aumento di LDH e bilirubina (7).

Le infezioni delle vie urinarie, frequenti a qualunque distanza dal trapianto, possono causare aumento della creatininemia, generalmente in assenza di oliguria, e prontamente reversibile con l'opportuna terapia antibiotica.

\section{Danno renale cronico}

Il danno cronico è graduale, progressivo nell'arco di mesi o anni, e le variazioni della creatininemia sono poco attendibili per valutare la funzione del trapianto nel lungo periodo perché possono essere dovute a variazioni della massa muscolare (1). Le formule per la stima del VFG consentono di predire il deterioramento della funzione del rene trapiantato meglio della sola creatininemia, soprattutto per valori di VFG tra 30 e $70 \mathrm{ml} /$ min, intervallo nel quale la creatinemia ha invece scarso valore predittivo (8). Nei trapiantati che presentano segni di disfunzione renale o proteinuria a un anno dal trapianto, il rischio di progressione all'insufficienza renale è aumentato e potrebbe quindi essere utile intensificare il ritmo dei controlli. Recentemente Kasiske ha messo a punto un modello per stimare, nei riceventi di trapianto da donatore cadavere, il rischio di perdere il rene trapiantato entro 5 anni in base a caratteristiche del paziente e del donatore ed al eVFG a una settimana o a un anno dall'intervento (9). Il calcolatore per la stima del rischio è disponibile online (www.txscores.org).

In passato il progressivo declino del VFG era il più delle volte attribuito a "rigetto cronico" con evidente riferimento all'eziologia immunologica del danno. Successivamente è stato introdotto il termine "chronic allograft nephropathy” (CAN): quadro istologico di fibrosi interstiziale, atrofia tubulare, alterazioni microvascolari e sclerosi glomerulare conseguenti sia a fattori immunologici sia a fattori non immunologici. Il termine CAN è stato poi spesso utilizzato per indicare genericamente l'entità clinica "disfunzione cronica del trapianto" come se corrispondesse a una precisa entità patologica. 
TABELLA I - INDICAZIONI ALL'ESECUZIONE DELLA BIOPSIA DEL RENE TRAPIANTATO SECONDO LE LINEE GUIDA K-DIGO 2009

Incremento persistente, non spiegato della creatininemia
Rigetto acuto, prima del trattamento ma a condizione di non ritardarne l'inizio
Mancato ritorno della creatininemia ai valori basali dopo il trattamento di un episodio di rigetto acuto
Ogni 7-10 giorni in caso di ritardata ripresa funzionale del trapianto (DGF = delayed graft function)
Funzione renale 1-2 mesi dopo il trapianto peggiore di quella attesa
Comparsa di proteinuria o proteinuria non spiegata $\geq 3 \mathrm{~g} / \mathrm{g}$ creatinina o $\geq 3 \mathrm{~g}$ al dì
Possibile recidiva di nefropatia di base suscettibile di trattamento
Nella colonna di destra è riportata la "forza" delle raccomandazioni.
$1 \mathrm{C}=$ raccomandazione scarsamente dimostrata, il cui effetto può essere sostanzialmente diverso dall'atteso.
2C = consiglio scarsamente dimostrato, il cui effetto può essere sostanzialmente diverso dall'atteso.
2D = consiglio molto scarsamente dimostrato: l'effetto atteso è molto incerto e spesso diverso da quello reale.

Da alcuni anni si preferisce quindi parlare di "chronic allograft injury" (CAI), per sottolineare la molteplicità delle possibili lesioni istologiche che possono e devono essere riconosciute dall'istologo e dei fattori di danno dell'organo (ipertensione, nefrotossicità cronica da inibitori delle calcineurine, rigetto, infezione da BKV, recidiva della nefropatia di base, nefropatia "de novo" ecc.) (10). La biopsia è quindi il gold standard per la valutazione del trapianto. Tuttavia le linee guida K-DIGO la consigliano solo in poche circostanze (Tab. I) e raccomandano l'esecuzione di trials clinici controllati e randomizzati per capire se i benefici delle biopsie protocollari siano o meno superiori ai rischi (1).

Per il nefrologo che segue in ambulatorio l'andamento della funzione del rene trapiantato queste considerazioni si traducono nella necessità di un rigoroso controllo di tutti i fattori correggibili di danno renale cronico quali l'ipertensione, la dislipidemia, il diabete, le infezioni e nell'attenzione ai livelli ematici dell'inibitore delle calcineurine. Il rapporto rischio/beneficio del passaggio a schemi di terapia immunosoppressiva senza inibitore della calcineurina è ancora incerto e la modifica va eventualmente concordata con il Centro Trapianti tenendo conto dell'entità della proteinuria, del VFG e - se possibile - del quadro istologico.

Nello studio di registro di Briganti su 1505 pazienti con biopsia sia del rene nativo sia del trapianto, la recidiva della nefropatia di base risultava la terza causa di perdita della funzione a 10 anni dal trapianto, dopo la morte del paziente con trapianto funzionante e la CAN (11). Frequenza, tempi, modalità di comparsa e impatto sulla funzione variano a seconda della patologia di base. La riduzione del VFG e/o la comparsa o il peggioramento di proteinuria costituiscono generalmente il primo elemento di sospetto cui devono seguire l'esame del sedimento urinario, per la ricerca di microematuria e cilindruria, eventuali indagini specifiche a seconda della nefropatia di base (complemento, ANCA, anti-MBG, piastrine, LDH) e la biopsia.

Maggiore incremento della creatininemia nel tempo e maggior rischio di rigetto acuto tardivo sono stati osservati nei pazienti che non seguono lo schema di terapia immunosoppressiva prescritto $(12,23)$. La non compliance riguarderebbe il $22-23 \%$ dei pazienti già dal primo anno dopo il trapianto $(12,13)$ ed è quindi importante che in occasione dei controlli periodici, il nefrologo ricordi al paziente di assumere regolarmente la terapia e ricerchi anche la collaborazione dei familiari.

In conclusione, l'aumento della creatininemia in un paziente trapiantato, anche se riscontrato a un controllo di routine e in assenza di sintomi, non deve mai essere sottovalutato.

\author{
Indirizzo degli Autori: \\ Gianna d'Adamo, MD \\ U.O.C. Nefrologia e Dialisi \\ Ospedale S. Spirito \\ ASL Roma/E \\ Lungotevere in Sassia 1 \\ 00193 Roma \\ giannadadamo@tiscalinet.it
}




\section{Bibliografia}

1. Kidney disease: Improving Global Outcomes (KDIGO) Transplant Work Group. KDIGO clinical practice guideline for the care of kidney transplant recipients. Am J Transplant 2009; 9(Suppl 3): S1-157.

2. Kasiske BL, Vazquez MA, Harmon WE, et al. Recommendations for the Outpatient Surveillance of Renal Transplant Recipients. J Am Soc Nephrol 2000; 11(Suppl): S186.

3. Oppenheimer F, Garcì Garcì M, Lòpez Alba T, et al. Coordinating between the renal transplant unit and the non transplant nephrology department. Nefrologia 2009; 29 (Suppl 1): S72-77.

4. Molitoris BA, Levin A, Warnock DG, et al. Improving outcomes from acute kidney injury. J Am Soc Nephrol 2007; 18(7): 1992-4.

5. Hariharan S, McBride MA, Cherikh WS, et al. Post-transplant renal function in the first year predicts long-term kidney transplant survival. Kidney Int 2002; 62: 311-8.

6. Blanckaert K, De Vriese AS. Current recommendations for diagnosis and management of polyoma BK virus nephropathy in renal transplant recipients. NDT 2006; 21 : 3364-7.
7. Mauiyyedi S, Colvin RB. Pathology of kidney transplantation. In Morris PJ, ed. Kidney transplantation. Principles and Practice. Philadelphia: WB Saunders Company, 2001; 343-76.

8. Chapman JR, O'Connell PJ, Nanlivell BJ. Chronic renal allograft dysfunction. J Am Soc Nephrol 2005; 16: 3015-26.

9. Kasiske BL, Ispani AK, Snyder JJ, et al. A simple tool to predict outcomes after kidney transplant. Am J Kidney Dis 2010; 56: 947-60.

10. Solez K, Colvin RB, Racusen LC. Banff '05 Meeting Report: Differential Diagnosis of Chronic Allograft Injury and Elimination of Chronic Allograft Nephropathy (CAN). Am J Transplant 2007; 7: 518-26.

11. Briganti EM, Russ Gr, McNeil JJ, et al. Risk of renal allograft loss from recurrent glomerulonephritis. N Eng J Med 2002; 347: 103-09.

12. Vlaminck H, Maes B, Evers G, et al. Prospective study on late consequences of subclinical non-compliance with immunosuppressive therapy in renal transplant patients. Am J Transplant 2004; 4: 1509-13.

13. De Geest S, Borgermans L, Gemoets H, et al. Incidence, determinants, and consequences of subclinical noncompliance with immunosoppressive therapy in renal transplant recipients. Transplantation 1995; 59: 340-7. 